\title{
Distinct Profiles on Subjective and Objective Adherence Measures in Patients Prescribed Antidepressants
}

\author{
Hans Wouters ${ }^{1,2} \cdot$ Didi Rhebergen $^{3} \cdot$ Marcia Vervloet $^{4} \cdot$ Antoine Egberts $^{5,6} \cdot$ Katja Taxis $^{1} \cdot$ Liset van Dijk $^{1,4}$. \\ Helga Gardarsdottir ${ }^{5,6,7}$
}

Published online: 3 April 2019

(c) The Author(s) 2019

\begin{abstract}
Objective A recurrent observation is that associations between self-reported and objective medication adherence measures are often weak to moderate. Our aim was therefore to identify patients with different profiles on self-reported and objective adherence measures.

Study Design and Setting This was an observational study of 221 community pharmacy patients who were dispensed antidepressants. Adherence profiles were estimated with Latent Profile Analysis (LPA) using data on self-reported adherence (Medication Adherence Rating Scale) complemented with data on medication beliefs (perceived necessity and concerns measured with the Beliefs about Medicines Questionnaire) and data from objective adherence measures (electronic monitoring of medication taking and the Medication Possession Ratio calculated from pharmacy dispensing data).

Results 'Goodness-of-fit' statistics indicated the presence of three classes: "concordantly high adherent" (83\%, high adherence on all measures), "concordantly suboptimal adherent" (11\%, low adherence on all measures), and "discordant" (6\%, high self-reported adherence but lower adherence on objective measures).

Conclusion Most patients had concordant outcomes on self-reported and objective measures of adherence. A small discordant class had high self-reported but low objective adherence. LPA will enable sensitivity analyses in future studies, for example excluding patients from the discordant class.
\end{abstract}

Hans Wouters

j.wouters@umcg.nl

1 Department of Pharmacotherapy, Pharmacoepidemiology and Pharmacoeconomics (PTEE), Faculty of Science and Engineering, Groningen Research Institute of Pharmacy, University of Groningen, Groningen, The Netherlands

2 Department of General Practice and Elderly Care Medicine, University Medical Center Groningen, University of Groningen, Oostersingel, Building 50, P.O. Box 196, 9700 AD Groningen, The Netherlands

3 Department of Old Age Psychiatry, InGeest Specialized Mental Health Care, Amsterdam UMC location VU Medical Center, Amsterdam, The Netherlands
4 Nivel Netherlands Institute for Health Services Research, Utrecht, The Netherlands

5 Division of Pharmacoepidemiology and Clinical Pharmacology, Faculty of Science, Utrecht Institute for Pharmaceutical Sciences, Utrecht University, Utrecht, The Netherlands

6 Department of Clinical Pharmacy, Division Laboratory and Pharmacy, University Medical Center Utrecht, Utrecht, The Netherlands

7 Faculty of Pharmaceutical Sciences, University of Iceland, Reykjavík, Iceland 


\section{Key Points}

Using Latent Profile Analysis, this study found three distinct profiles on subjective and objective measures of medication adherence: a "concordantly high adherent" profile, a "concordantly suboptimal adherent" profile, and a "discordant" profile of high self-reported adherence but low objective adherence.

Latent Profile Analysis of adherence measures demonstrates that researchers and clinicians should not rely on a single adherence measure, but rather should conduct rigorous sensitivity analyses of data on multiple adherence measures.

\section{Introduction}

A recurrent observation in the medication adherence literature is that associations between different measures of adherence tend to be weak to moderate [1-3]. Available measures of medication adherence include subjective assessment methods such as questionnaires for self-reported adherence. Opposed to these are objective measures such as those inferred from pharmacy dispensing data and electronic monitoring of medication intake with a device. The co-existence of all these methods is explained by the fact that each method has its own strengths and limitations. Although pharmacy dispensing data are objective, they only provide information about whether a patient fills their prescriptions and not so much whether a patient actually took their medication. Electronic monitoring of medication intake informs more precisely whether a patient took their medication, but it is costly and less feasible as it requires installment of a device in a patient's home. Questionnaires are feasible and inexpensive but may be distorted by recall and social desirability bias.

Hitherto, studies about the associations of different measures of medication adherence have been conducted at the overall group level. However, the often observed weak to moderate associations between these measures warrant an examination of distinct patient populations with different profiles on adherence measures. Methods that enable an identification of different patient populations (i.e. 'classes') in a data-driven bottom-up manner may serve this goal. Here, we adopt one of these methods, called Latent Profile Analysis (LPA), to identify profiles on different measures of adherence. Compared to classifying individuals by face-validity or cluster-analysis, the LPA analysis is governed by so-called "goodness-of-fit" statistics. These provide an empirically based approach to assess the most likely number of patient classes with different adherence profiles. LPA or related approaches have been used to study amongst others adherence subtypes in hypertensive patients [4], depression subtypes among patients treated with citalopram [5], examination of the nosological status of generalized anxiety disorders versus dysthymic disorder [6], and course trajectories of unipolar depressive disorders [7].

In this study, we applied LPA to examine profiles on different adherence measures among patients who are prescribed antidepressants. These medications are frequently prescribed [8] and are an important therapeutic strategy for the long-term treatment of depression, anxiety, and other disorders [9]. At the same time, non-adherence to antidepressant therapy is substantial. Previous studies reported the incidence and prevalence of non-adherent patients to range from approximately one-third $[10,11]$ to half $[12,13]$ to about three in five [14] of the patients. Non-adherence to antidepressants is explained by previous findings showing depression to actually be a predictor of non-adherence [15]. Non-adherence to antidepressants is also a clinically important problem as it was previously found to be associated with an increased relapse risk [12]. Thus, like for other patient groups, proper measurement and detection of non-adherence among patients prescribed antidepressants is crucial. With regard to antidepressants, associations between different adherence measures were found to be mixed. Weak to moderate associations were found between self-reported adherence and electronic monitoring of medication intake $[13,14]$. Associations between self-reported adherence and adherence based on pharmacy dispensing data were moderate to strong [16]. Other researchers found weak associations between pill counts and plasma drug concentration [17].

Accordingly, using LPA the aim of the present study was to identify profiles on subjective and objective adherence measures among patients who were prescribed antidepressants.

\section{Methods}

\subsection{Study Design and Setting}

Between April 2011 and April 2012, patients were enrolled through five community pharmacies of the Utrecht Pharmacy Practice network for Education and Research (UPPER) that were located throughout the Netherlands [18]. All pharmacies had automated dispensing records that contained information about patients' sex, date of birth, dispensed medications, dispensing dates, type of prescriber, amount of medications dispensed, and prescribed dosages. The duration of antidepressant use was estimated by dividing the number of dispensed units by the prescribed number of units per day. All medications were coded according to 
the Anatomical Therapeutic Chemical (ATC) classification index [19]. The reporting of study findings followed the ESPACOMP Medication Adherence Reporting Guideline (EMERGE) for medication adherence studies [20] and the STrengthening the Reporting of OBservational studies in Epidemiology (STROBE) criteria [21].

\subsection{Participants}

Patients were eligible if they were $\geq 18$ years and if an antidepressant had been dispensed to them (selective serotonin reuptake inhibitors, ATC codes N06AB; non-selective monoamine-oxydase inhibitors, N06AF; monoamine-oxydase inhibitors, N06AG; and other antidepressants, N06AX).

Patients were not included if they were dispensed tricyclic antidepressants (TCAs). TCAs are increasingly prescribed by physicians for conditions other than depression [22], which are often not documented in the automated pharmacy dispensing records [9]. Non-adherence was not an exclusion criterion. Depending on the number of patients in a pharmacy who were dispensed antidepressants, every fourth or fifth patient was selected for participation. To prevent selection bias, patients were not selected by the pharmacist, but rather with the aid of the automated dispensing records by an impartial research assistant. Eligible patients received an invitation to participate in the study and a questionnaire about sociodemographic characteristics, medication use including previous use of antidepressants, disease characteristics, self-reported adherence, and medication perceptions. Patients were requested to return the completed questionnaire within 2 weeks. In case of non-response, they were sent three reminders by mail. The index date was defined as the date on which the signed written informed consent and the questionnaire were received by the study team. Subsequently, patients were asked to get their antidepressants dispensed in the Medication Event Monitoring System (MEMS) device during a period of 6 months (see below).

\subsection{Adherence to and Perceptions about Antidepressant Therapy}

Medication adherence was assessed by subjective and objective methods. The subjective measure included self-reported adherence as assessed with the Medication Adherence Rating Scale (MARS) [23]. The MARS was complemented by patients' "perceived necessity" and "perceived concerns" about antidepressants as assessed with the Beliefs about Medicines Questionnaire (BMQ). Although the BMQ perceptions of "perceived necessity" and "perceived concerns" are not measures of adherence, they are likely to reflect patients' willingness versus reluctance to take their medicines [23]. Objective measures included electronic monitoring of medication intake using the MEMS (Aardex, Zug, Switzerland) and the Medication Possession Ratio (MPR) calculated from pharmacy dispensing data (see Fig. 1 for the time window during which the data for different adherence measures were collected). In the literature, the authoritative $A B C$ taxonomy of adherence distinguishes between three essential adherence phases, namely "initiation", "implementation", and "persistence" [20, 24]. Based on the ABC taxonomy, the focus of the present study was mainly on the adherence phase of "implementation".

The MARS consists of five items to assess the following non-adherence behaviors: forget to take antidepressants, altering the dose, stop using antidepressants for a while, decide to skip a dose, and taking less antidepressants than prescribed. Patients had to rate each item on a 5-point scale ( $1=$ always, $5=$ never $)$. Subsequently, these ratings were summated to obtain a total score with higher scores being indicative of better adherence (range 5-25). The BMQ consists of items that are rated on a 5-point scale $(1=$ completely disagree, $5=$ completely agree). Consensus has been reached that these items measure "perceived necessity," e.g.: "My health at present depends on my antidepressants" (five items, range 5-25), and "perceived concerns," e.g.: "I am afraid of the side effects of antidepressants" (six items, range 6-30).

The MEMS devices registered times and dates of occasions on which patients had opened the medication vial, thereby enabling the monitoring of patients' daily medication intake. All data from the MEMS devices were downloaded after the data collection period. From these MEMS data, we assessed the percentage of therapy days on which antidepressants were taken, i.e.: $100-$ ((number of days missed/total number of MEMS days $) \times 100$ ). Thus, higher percentages reflected better adherence.

Information on all dispensed medication to the patients was extracted from the automated dispensing records of the pharmacies. "Treatment episodes" were determined for each patient in the same way as described elsewhere [25]. Subsequently, we estimated the MPR for the last treatment episode prior to the index date or the "number of days' supply between the first prescription within the treatment episode and the index date" divided by "the number of days elapsing between the first prescription within the treatment episode and the index date". Higher MPR percentages reflected better adherence.

\subsection{Statistical Analysis}

Patients' demographic and clinical characteristics were summarized with descriptive statistics. MARS and BMQ scores were recalculated as percentages to facilitate mutual comparisons between these measures of adherence with the MPR and MEMS that were already calculated as percentages.

Using Latent Profile Analysis (LPA), we empirically examined patient populations (i.e. 'classes') with different 
Fig. 1 Time window of data from different adherence measures being collected

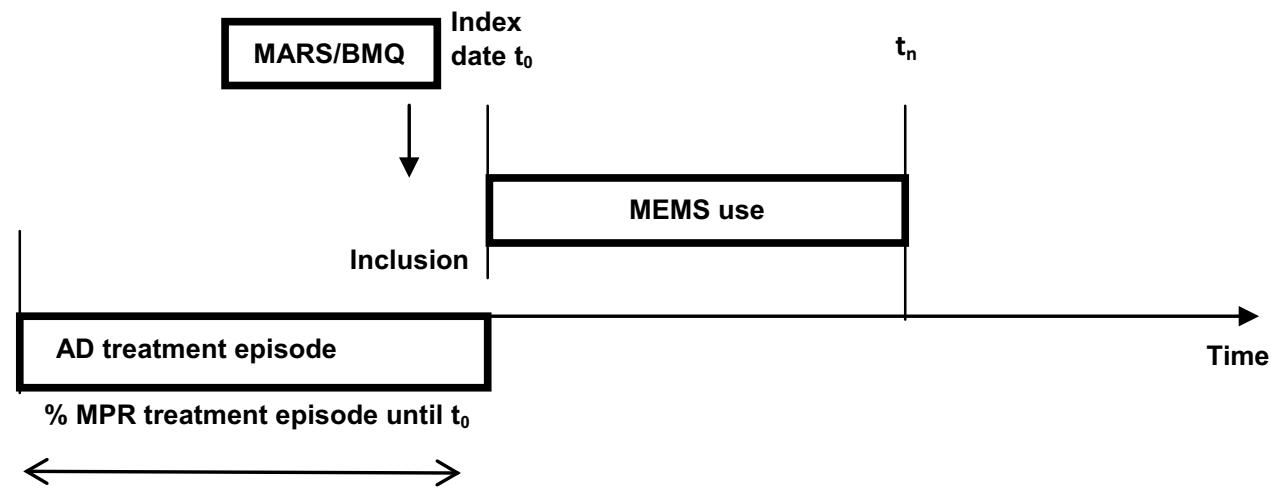

adherence profiles on the indicator variables of self-reported adherence (MARS), electronic monitoring of medication intake (MEMS), and adherence inferred from pharmacy dispensing data (MPR), as well as self-reported "perceived necessity" and "perceived concerns" (BMQ). The BMQ measures were added as it was previously shown that increasing the number of indicator variables improved the stability of the LPA parameter estimates [26]. Identification of profiles occurred in a successive forward manner. First, we estimated an LPA model with a single class to which all patients were assigned. This corresponds to a standard statistical analysis of the total group. Subsequently, successive models were estimated in which "latent classes" were progressively added, one at a time. The most likely number of classes were those from the model with optimal statistical "goodness of fit". For LPA, various "goodness of fit" statistics are available. As recommended previously [27], we inspected the Bayesian Information Criterion (BIC) and the Bootstrapped Likelihood Ratio Test (BLRT). In addition, we also inspected the Entropy. For the BIC, smaller values are indicative of better "goodness of fit". The BLRT tests whether the current model with $\mathrm{n}$ latent classes has a better "goodness of fit" than the preceding model with $n-1$ latent classes. Improvement was deemed significant if the associated $p$ value was $<0.05$. The Entropy ranges from 0 to 1 and is an indication of the quality of classification of individuals into classes. Entropy values $>0.8$ are acceptable. To identify clinically relevant classes, alongside goodness of fit, classes had to contain at least $5 \%$ of study patients [6, 7]. Because LPA is susceptible to converging on local rather than global solutions, we used multiple random starting values in estimating the LPA models (500 repeats with 20 final optimizations) [7]. Furthermore, the MARS, MEMS, and MPR variables seemed to be right-censored (with the distributions lacking a right tail beyond the maximum score) and were therefore specified in the model accordingly.

In the primary LPA analysis, we included patients with complete data on all adherence measures $(N=131)$. In a secondary analysis, the LPA analysis was repeated with all 221 patients of whom 90 who had complete data on the
MARS, MPR, and BMQ measures but missing values on the MEMS. In LPA, missing values are dealt with by estimating parameters with maximum likelihood. After identification of latent classes, individuals were assigned to the most likely class using estimated class probabilities. The LPA analysis was conducted using Mplus (version 7.4). Individuals from different latent classes of adherence measures were then compared on demographic and clinical characteristics in descriptive analyses using SPSS (version 24).

\section{Results}

\subsection{Participants' Characteristics}

Of the 871 eligible patients who were approached, 221 consented to participate. Three-quarters of patients were women, more than half were living with a partner, about half of the patients attained an intermediate educational level, and most patients were treated with paroxetine. About half of the patients were treated for depression as opposed to the remaining patients who were treated for anxiety, for depression with concomitant anxiety, or other disorders (Table 1).

\subsection{Profiles of Adherence Measures}

In the primary LPA analysis of patients with complete data on all adherence measures $(N=131)$, "goodness-of-fit" statistics (BIC, BLRT, Entropy) indicated that three classes of patients were most likely (Table 2). After plotting the mean scores of these three classes of patients on the adherence measures, the classes could be interpreted as a concordantly high adherent class (83\%), which scored high on all adherence measures and which had high BMQ necessity and low BMQ concerns; a concordantly suboptimal adherent class (11\%) with lower scores on all adherence measures and lower BMQ necessity but comparable BMQ concerns; and a discordant class (6\%) with high self-reported adherence on the MARS but lower adherence on objective measures of MEMS and MPR while exhibiting high BMQ necessity 
Table 1 Demographic and clinical characteristics of participants $(N=221)$

\begin{tabular}{lc}
\hline Characteristics & Statistic \\
\hline Demographic characteristics & \\
$N(\%)$ women & $162(73.3)$ \\
$M(\mathrm{SD})$ age, years & $53.0(14.6)$ \\
$N(\%)$ with spouse/partner & $117(52.9)$ \\
$N(\%)$ educational level & \\
Low & $56(25.4)$ \\
Intermediate & $112(50.6)$ \\
High & $53(24)$ \\
Clinical characteristics & \\
$N$ (\%) type of antidepressant & \\
Paroxetine & $84(38)$ \\
Fluoxetine & $13(6)$ \\
Fluvoxamine & $5(2)$ \\
Sertraline & $9(4)$ \\
Citalopram & $42(19)$ \\
Venlafaxine & $35(16)$ \\
Escitalopram & $13(6)$ \\
Other/unknown & $20(9)$ \\
$N(\%)$ diagnosis & \\
Depression & $109(49)$ \\
Anxiety & $49(22)$ \\
Depression and concomitant anxiety & $48(22)$ \\
Other condition & $15(7)$ \\
\hline
\end{tabular}

and low BMQ concerns (Fig. 2). Similar results were found in the secondary analysis in which the LPA analysis was repeated for all 221 patients including those 90 patients with complete data on the MARS, MPR, and BMQ measures but with missing values on the MEMS.

\subsection{Descriptive Analyses of Profiles}

Table 3 displays the demographic and clinical characteristics of the three classes of patients with distinct adherence profiles. Compared to the concordantly high adherent class and concordantly suboptimal adherent class, patients in the discordant class were more often women, were younger, were less often living with a partner, less often had a high educational level, were less often using paroxetine, and had a depression diagnosis more often.

\section{Discussion}

We observed three distinct profiles on subjective and objective adherence measures in this sample of patients who were prescribed antidepressants. We found a large, concordantly high adherent class that was adherent on all measures and a concordantly suboptimal adherent class that had lower adherence on all measures. Interestingly, we also identified a small discordant class with high selfreported adherence but lower adherence on objective measures of adherence and high "perceived necessity" and low "perceived concerns".

Our findings are important as non-adherence is prevalent in patients treated with antidepressants [28]. We found that almost one in five patients could be considered non-adherent (i.e., the patients from the "concordantly suboptimal adherent" and the "discordant" classes together). Previously, the incidence and prevalence of non-adherence were found to range from about one-third $[10,11]$ to half $[12,13]$ to three in five [14] of the patients. The present findings, and especially the finding of a discordant class of patients with high self-reported adherence but lower objectively measured adherence, are consistent with findings from a scoping review of studies in which the MEMS was compared with other adherence measures [29] as well as with findings from previous individual studies [1-3].

The identification of different adherence profiles and the detection of the discordant adherence class was enabled because adherence was measured using both subjective (selfreported MARS) and objective measures (MEMS and MPR through pharmacy dispensing data). In studies, assessment of adherence is often limited to a single method particularly self-report. Given the advantages and disadvantages of different adherence measures, multiple methods to assess therapy adherence are complementary. The MPR is primarily a measure of long-term persistence to a particular medication or a class of medications while electronic monitoring is a measure of dosing regimen implementation to better understand patient habit of medication taking. Adoption of multiple assessment methods of adherence in research and clinical practice is likely to maximize accuracy $[3,15]$. Our assessment of medication adherence with both subjective and objective methods is therefore the most important strength of this study.

A strength of the LPA is that it provides insight into the presence of relevant subpopulations of patients as was previously examined for hypertensive patients with regard to adherence to medications, diet, exercise, smoking, and blood pressure [4]. LPA provides a more formal approach to assess the most likely number of classes compared to methods such as cluster-analysis or classifying individuals by face-validity, because it is governed by "goodness-of-fit" statistics.

For clinical practice, these findings have two implications. First, clinicians should not solely rely on self-reported therapy adherence. Rather, they should seek verification of patients' answers from for example pharmacy dispense data and the MPR calculated therefrom. Where there is not a closed pharmacy system such as with the Department of Veterans Affairs Health Care System in the USA or the system 
Table 2 Parameters of fit of latent profile analysis $(N=131)$

\begin{tabular}{|c|c|c|c|c|c|c|c|c|c|c|}
\hline \multirow[t]{2}{*}{ Class } & \multirow{2}{*}{$\begin{array}{l}\text { Maximum likeli- } \\
\text { hood }\end{array}$} & \multirow[t]{2}{*}{$\mathrm{BIC}$} & \multirow[t]{2}{*}{ Entropy } & \multicolumn{3}{|l|}{ BLRT test } & \multicolumn{4}{|c|}{$\%$ of individuals in class } \\
\hline & & & & $\begin{array}{l}2 \times \text { LL differ- } \\
\text { ence }\end{array}$ & $d f$ & $p$ & 1 & 2 & 3 & 4 \\
\hline 1 & - & - & - & - & - & - & - & - & - & - \\
\hline 2 & -2265 & 4609 & 0.93 & 57.6 & 6 & 0.17 & 85 & 15 & - & - \\
\hline 3 & -2244 & 4596 & 0.97 & 42.2 & 6 & 0.048 & 11 & 6 & 83 & - \\
\hline 4 & -2235 & 4606 & 0.96 & 18.7 & 6 & 0.49 & 11 & 6 & 4 & 79 \\
\hline
\end{tabular}

BLRT Bootstrapped Likelihood Ratio Test, BIC Bayesian Information Criterion

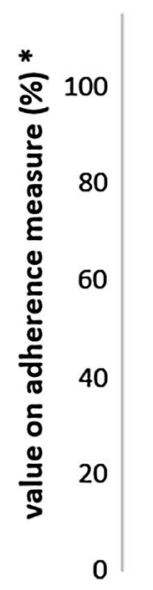

Fig. 2 Scoring profiles of patient classes on adherence measures. *Values on adherence measures are expressed as percentages to facilitate mutual comparisons between different adherence measures.
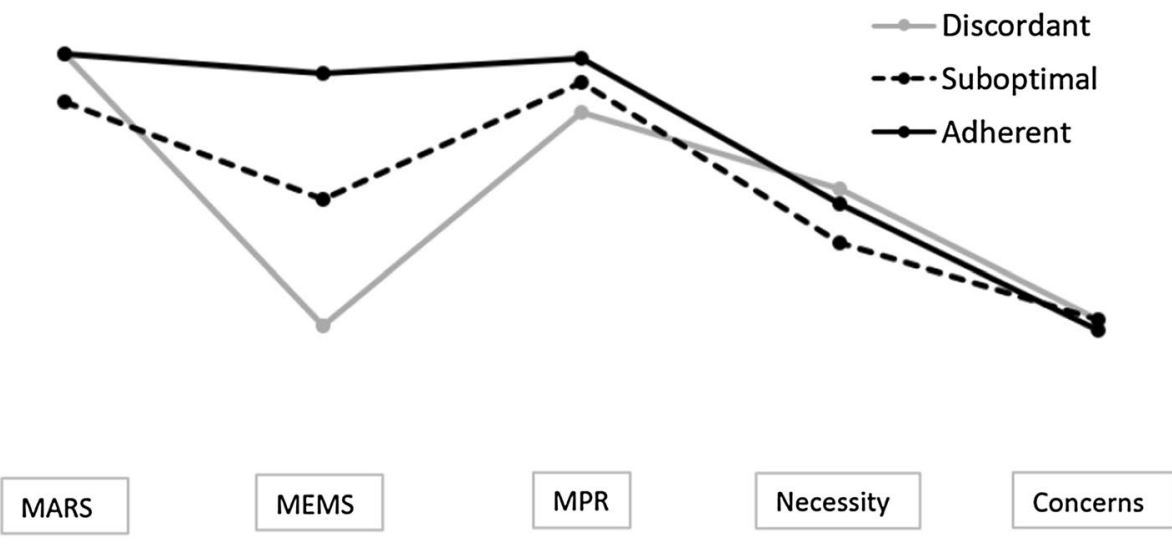

MARS Medication Adherence Rating Scale, MEMS Medication Event Monitoring System, MPR Medication Possession Ratio

Table 3 Demographic and clinical characteristics of patients from three adherence profiles

\begin{tabular}{lccc}
\hline Characteristics & \begin{tabular}{l} 
Adherence profiles \\
\cline { 2 - 4 }
\end{tabular} & $\begin{array}{l}\text { Concordant high adherent } \\
(N=109)\end{array}$ & $\begin{array}{l}\text { Concordant suboptimal adherent } \\
(N=15)\end{array}$ \\
\hline Demographic characteristics & & & Discordant $(N=7)$ \\
$N(\%)$ women & $85(78)$ & $10(67)$ & $7(100)$ \\
$M(\mathrm{SD})$ age, years & $51.3(13.8)$ & $53.1(13.1)$ & $42.9(14.8)$ \\
$N(\%)$ with spouse/partner & $79(73)$ & $13(87)$ & $4 /(57)$ \\
$N(\%)$ with high educational level & $33(30)$ & $6(40)$ & $1(14)$ \\
Clinical characteristics & & $8(53)$ & $1(14)$ \\
$N(\%)$ with paroxetine use & & $4(57)$ \\
$N(\%)$ with depression diagnosis ${ }^{\mathrm{b}}$ & $41(38)$ & $2(13)$ & \\
\hline
\end{tabular}

${ }^{a}$ Paroxetine vs. fluoxetine, fluvoxamine, sertraline, citalopram, venlafaxine, escitalopram, and other/unknown

${ }^{\mathrm{b}}$ Depression vs. anxiety, depression and concomitant anxiety, or other condition

of community pharmacies in the Netherlands, electronic monitoring of medication intake could be used to verify self-reported therapy adherence. Second, in line with the previous implication, implementation of feasible electronic monitoring of medication adherence in clinical practice is of paramount importance and should be expedited.

For research, adoption of multiple assessment methods of adherence enables investigation of the following 
opportunities. First, it would be worthwhile to conduct a sensitivity analysis in which a discordant or otherwise atypical class as revealed by LPA may be left out, after which the primary analysis is repeated. Second, more research is needed about the prevalence and characteristics of the discordant class. The discordant pattern may represent distortions of self-reported adherence by social desirability, perhaps recall problems owing to cognitive impairment, or the intention to be adherent as in self-report versus actual non-adherence according to electronic monitoring or a combination of these possible explanations. It may therefore be valuable to conduct qualitative interviews [30] with individuals to examine reasons for such a discordance.

A number of methodological issues deserve to be addressed. First of all, although our sample size was moderate, it comprised more than 100 participants. Previously, a Monte Carlo simulation study demonstrated that a sample size of 100 participants was sufficiently large to conduct an LPA analysis, taking into consideration also that electronic monitoring of medication intake constitutes a high-quality measure [26]. Having said this, replications in larger samples could still be fruitful, especially with regard to comparing identified classes on demographic and clinical characteristics. Second, with regard to the sampling, selection bias may have occurred in the sense that adherent patients were more likely to participate. As a result, the sizes of the concordant suboptimal adherent and discordant classes may have been underestimated. However, it should also be noted that even so a substantial number of participating patients still had a lower adherence according to MEMS. Third, with regard to the adherence assessments, potential instrumentation effects may have occurred, for example monitoring of medication intake with the MEMS may have improved adherence at least in some patients. Given that the MPR and MARS were assessed before the installment of the MEMS in patients' homes, these measures were not affected by possible instrumentation effects of the MEMS. Naturally, to detect instrumentation effects, future research would need to adopt a factorial design where half of the participants would have all adherence measures monitored during the same time period while others would have not. Fourth, other adherence measures as inferred from the MEMS are worthwhile considering. Fifth, with regard to the ABC taxonomy $[20,24]$ of successive phases of adherence, namely "initiation," "implementation," and "persistence," it is noteworthy that our study was mainly focused on the "implementation" phase of antidepressant use. We had no data on prescriptions and could therefore not study the "initiation" or earlyuse phase in which a patient fills a prescription. Incorporating the "initiation" phase could therefore be valuable to examine if there are yet other distinct profiles on subjective and objective adherence measures among newly diagnosed patients or patients in the early treatment phase.
Taken together, we found distinct adherence profiles among patients who were prescribed antidepressants. This emphasizes the need to measure adherence through multiple subjective and objective methods. Gaining more insights into the prevalence and characteristics of these different classes and in particular the discordant class is warranted.

Acknowledgements The authors would like to thank the pharmacies and participants for their time and efforts.

\section{Compliance with Ethical Standards}

Funding The study was financially supported by a VENI grant from the Netherlands Organization for Health Research and Development (ZonMw) awarded to HG (Grant Number 91611147). LvD reports funding for studies not related to this manuscript from Astra Zeneca, Pfizer, and AbbVie.

Conflict of interest The authors report no other conflicts of interest.

Ethical standards All procedures performed in this study were in accordance with the ethical standards of the medical ethics committee of the University Medical Center of Utrecht, which approved the study, and with the 1964 Helsinki declaration and its later amendments or comparable ethical standards.

Informed consent Written informed consent was obtained from all individual participants who were included in the study.

Open Access This article is distributed under the terms of the Creative Commons Attribution-NonCommercial 4.0 International License (http://creativecommons.org/licenses/by-nc/4.0/), which permits any noncommercial use, distribution, and reproduction in any medium, provided you give appropriate credit to the original author(s) and the source, provide a link to the Creative Commons license, and indicate if changes were made.

\section{References}

1. Oberguggenberger AS, Sztankay M, Beer B, Schubert B, Meraner V, Oberacher $\mathrm{H}$, et al. Adherence evaluation of endocrine treatment in breast cancer: methodological aspects. BMC Cancer. 2012;12:474.

2. Wetzels GE, Nelemans PJ, Schouten JS, van Wijk BL, Prins MH. All that glisters is not gold: a comparison of electronic monitoring versus filled prescriptions-an observational study. BMC Health Serv Res. 2006;6:8.

3. Shi L, Liu J, Fonseca V, Walker P, Kalsekar A, Pawaskar M. Correlation between adherence rates measured by MEMS and self-reported questionnaires: a meta-analysis. Health Qual Life Outcomes. 2010;8:99.

4. Trivedi RB, Ayotte BJ, Thorpe CT, Edelman D, Bosworth HB. Is there a nonadherent subtype of hypertensive patient? A latent class analysis approach. Patient Prefer Adherence. 2010;4:255-62.

5. Ulbricht CM, Rothschild AJ, Lapane KL. The association between latent depression subtypes and remission after treatment with citalopram: a latent class analysis with distal outcome. J Affect Disord. 2015;188:270-7.

6. Rhebergen D, van der Steenstraten IM, Sunderland M, de Graaf R, Ten Have M, Lamers F, et al. An examination of generalized anxiety disorder and dysthymic disorder by latent class analysis. Psychol Med. 2014;44:1701-12. 
7. Rhebergen D, Lamers F, Spijker J, de Graaf R, Beekman AT, Penninx BW. Course trajectories of unipolar depressive disorders identified by latent class growth analysis. Psychol Med. 2012;42:1383-96.

8. Zorginstituut Nederland. 2016. http://www.gipdatabank.nl. Accessed 22 Oct 2018.

9. Gardarsdottir H, Heerdink ER, van Dijk L, Egberts AC. Indications for antidepressant drug prescribing in general practice in the Netherlands. J Affect Disord. 2007;98:109-15.

10. Sirey JA, Bruce ML, Alexopoulos GS, Perlick DA, Friedman SJ, Meyers BS. Stigma as a barrier to recovery: Perceived stigma and patient-rated severity of illness as predictors of antidepressant drug adherence. Psychiatr Serv. 2001;52:1615-20.

11. Brook OH, van Hout HP, Stalman WA, de Haan M. Nontricyclic antidepressants: predictors of nonadherence. J Clin Psychopharmacol. 2006;26:643-7.

12. ten Doesschate MC, Bockting CL, Schene AH. Adherence to continuation and maintenance antidepressant use in recurrent depression. J Affect Disord. 2009;115:167-70.

13. Lee MS, Lee HY, Kang SG, Yang J, Ahn H, Rhee M, et al. Variables influencing antidepressant medication adherence for treating outpatients with depressive disorders. J Affect Disord. 2010;123:216-21.

14. Bogner HR, de Vries HF, O’Donnell AJ, Morales KH. Measuring concurrent oral hypoglycemic and antidepressant adherence and clinical outcomes. Am J Manag Care. 2013;19:e85-92.

15. Osterberg L, Blaschke T. Adherence to medication. N Engl J Med. 2005;353:487-97.

16. Rickles NM, Svarstad BL. Relationships between multiple selfreported nonadherence measures and pharmacy records. Res Social Adm Pharm. 2007;3:363-77.

17. Woldu H, Porta G, Goldstein T, Sakolsky D, Perel J, Emslie G, et al. Pharmacokinetically and clinician-determined adherence to an antidepressant regimen and clinical outcome in the TORDIA trial. $\mathrm{J}$ Am Acad Child Adolesc Psychiatry. 2011;50:490-8.

18. Koster ES, Blom L, Philbert D, Rump W, Bouvy ML. The Utrecht Pharmacy Practice network for Education and Research: a network of community and hospital pharmacies in the Netherlands. Int J Clin Pharm. 2014;36:669-74.

19. WHO Collaborating Centre for Drug Statistics Methodology. Anatomical Therapeutic Chemical (ATC) Classification Index. Available at: http://www.whocc.no/atc_ddd_index/. Accessed 22 Oct 2018.
20. De Geest S, Zullig LL, Dunbar-Jacob J, Helmy R, Hughes DA, Wilson IB, et al. ESPACOMP Medication Adherence Reporting Guideline (EMERGE). Ann Intern Med. 2018;169:30-35.

21. von Elm E, Altman DG, Egger M, Pocock SJ, Gotzsche PC, Vandenbroucke JP, et al. The Strengthening the Reporting of Observational Studies in Epidemiology (STROBE) statement: guidelines for reporting observational studies. J Clin Epidemiol. 2008;61:344-9.

22. Noordam R, Aarts N, Verhamme KM, Sturkenboom MC, Stricker $\mathrm{BH}$, Visser LE. Prescription and indication trends of antidepressant drugs in the Netherlands between 1996 and 2012: a dynamic population-based study. Eur J Clin Pharmacol. 2015;71:369-75.

23. Horne R, Weinman J. Patients' beliefs about prescribed medicines and their role in adherence to treatment in chronic physical illness. J Psychosom Res. 1999;47:555-67.

24. Vrijens B, De Geest S, Hughes DA, Przemyslaw K, Demonceau J, Ruppar T, et al. A new taxonomy for describing and defining adherence to medications. Br J Clin Pharmacol. 2012;73:691-705.

25. Gardarsdottir H, Souverein PC, Egberts TC, Heerdink ER. Construction of drug treatment episodes from drug-dispensing histories is influenced by the gap length. J Clin Epidemiol. 2010;63:422-7.

26. Wurpts IC, Geiser C. Is adding more indicators to a latent class analysis beneficial or detrimental? Results of a Monte-Carlo study. Front Psychol. 2014;5:920.

27. Nylund KL, Asparouhov T, Muthén BO. Deciding on the number of classes in latent class analysis and growth mixture modeling: a Monte Carlo simulation study. Struct Equ Model. 2007;14:535-69.

28. Pampallona S, Bollini P, Tibaldi G, Kupelnick B, Munizza C. Patient adherence in the treatment of depression. Br J Psychiatry. 2002;180:104-9.

29. El Alili M, Vrijens B, Demonceau J, Evers SM, Hiligsmann M. A scoping review of studies comparing the medication event monitoring system (MEMS) with alternative methods for measuring medication adherence. Br J Clin Pharmacol. 2016;82:268-79.

30. Britten N. Patients' ideas about medicines: a qualitative study in a general practice population. Br J Gen Pract. 1994;44:465-8. 